\title{
Label-free detection of DNA hybridization based on hydration- induced tension in nucleic acid films
}

\author{
Johann Mertens ${ }^{1}$, Celia Rogero ${ }^{2}$, Montserrat Calleja ${ }^{1}$, Daniel Ramos ${ }^{1}$, Jose Angel \\ Martín-Gago $^{2,3}$, Carlos Briones ${ }^{2}$ and Javier Tamayo ${ }^{1, \#}$ \\ ${ }^{1}$ Bionanomechanics Lab (IMM-CNM), CSIC 28760 Tres Cantos, Madrid, Spain \\ ${ }^{2}$ Centro de Astrobiología (CSIC-INTA). 28850 Torrejón de Ardoz, Madrid, Spain. \\ ${ }^{3}$ Instituto de Ciencia de Materiales de Madrid (CSIC). Cantoblanco, 28049 Madrid, \\ Spain.
}

Here we show that adsorption of water on highly-packed self-assembled monolayers (SAMs) of single stranded (ss) DNA has an extraordinary effect on the intermolecular interactions. We have followed the process by measuring the nano-scale bending that a silicon microcantilever, on which the ssDNA monolayer is attached, experiences under controlled relative humidity. More importantly, the hydration-induced tension undergoes dramatic changes when the monolayer interacts with either complementary or single mismatched ssDNA targets. The analysis of the results suggests that the tension of the nucleic acid films is mainly governed by the hydration forces originated in the intermolecular channels. The discovered phenomena open the door to the development of a novel label-free DNA biosensor with specificity to single mutations and a sensitivity of at least ten times higher than the label-dependent DNA microarrays.

The change in the properties of water at the nanoscale is crucial in the structure and intermolecular interactions of biological assemblies ${ }^{1}$. Self-assembled monolayers (SAMs) of single-stranded (ss) DNA probes inmobilised on solid supports is the base of a variety of biosensors and nanotechnological devices ${ }^{2-4}$. At high packing conditions, ssDNA molecules stand-up originating intermolecular channels. Although the confinement of water in the sub-nanometer channels should play a fundamental role in the intermolecular interactions, it has received little attention. Advances in nanotechnology, in particular those based in micro- and nanomechanical sensors ${ }^{5-16}$, can potentially be used to analyze the role played by water molecules in the 
macromolecular interactions ${ }^{17-19}$ leading to a new a generation of ultrasensitive biosensors.

The experimental set-up developed to study the forces induced by the water confinement in DNA monolayers, as well as all the experimental details, are described in the Methods and the Supplementary Information. Basically, we form a dense SAM of a thiol-modified 16-mer ssDNA on the gold-coated side of a silicon microcantilever ${ }^{12,2,3}$. The DNA sequence was selected from the human BRCA1 gene that can contain mutations involved in breast cancer ${ }^{20}$. The microcantilever is placed in a humidity chamber, in which the relative humidity (r.h.) is controlled by the ratio between dry and humid nitrogen. A modification of the forces between the anchored DNA molecules translates into a nanomechanical motion (bending) of the cantilever, which is measured by a scanning optical technique recently developed ${ }^{21}$.

Fig. 1 shows the surface stress variation as a function of the relative humidity in a hydration/dehydration cycle for a ssDNA sensitised cantilever. We distinguish three stages in the hydration curve. Stage I occurs during the adsorption of the first water molecules, up to $r . h=5-20 \%$, and it is characterized by a sharp rise of the surface stress (tensile stress) of about $40-70 \mathrm{mN} / \mathrm{m}$. Higher hydration of the ssDNA monolayer leads to a significant decrease of the surface stress that extends up to a r.h. value of $50-70 \%$. In this region of the curve, referred to as stage II, the compressive variation of the surface stress is of about $150-200 \mathrm{mN} / \mathrm{m}$. In the region of higher hydration up to reaching the wet state, the surface stress slightly decreases with r.h. (stage III). During dehydration, the surface stress shows little variations up to a critical value of r.h. of about 20\%, after which it rapidly increases returning to the initial value of the hydration/dehydration cycle. As a reference, the hydration/dehydration loop for the gold-coated cantilever prior to the ssDNA monolayer assembly is plotted. The surface stress shows almost a flat response when the r.h. is varied. This demonstrates that the significant hydration-dependence of the ssDNA surface stress arises from the interaction between the water and the anchored DNA molecules. Notice that the surface stress at the dry state is set to as the zero reference as the study is focused on the hydration-induced surface stress.

The behaviour described above was exclusively found for immobilization times of the ssDNA of 24-48 hours. For shorter immobilization times, the surface stress did not exhibit a noticeable dependence on the r.h. (see Supplementary Information). This suggests that the observed phenomena requires the formation of a standing up SAM, in which the SSDNA molecules are anchored to the surface only via their terminal thiol groups $^{2,3}$. Analysis by X-ray photoelectron spectroscopy (XPS) indicates the ssDNA 
monolayers used in this work have a surface density of about $4 \times 10^{13}$ molecules per $\mathrm{cm}^{2}$ with most of the molecules standing-up (see Supplementary Information). The high packing density of the ssDNA monolayer drives its behaviour as a molecular membrane, in which the molecules cooperatively adopt a conformation in response to interactions brought by the insertion of new molecules into the membrane. Assuming that the ssDNA molecules behave as cylinders with a diameter of $1.3 \mathrm{~nm}$ and adopt a hexagonal packing, the DNA membrane hosts intermolecular channels with a diameter of about $0.8 \mathrm{~nm}$ (see cartoons in Fig. 1). The initial hydration (stage I), is related to the binding of water molecules to the surface of the ssDNA strands. This process leads to the formation of the DNA hydration shells, and it is driven by the avidity of water to form hydrogen bonds with the phosphate groups, the sugar oxygen atoms, and the lateral polar groups of the nucleobases ${ }^{22,23}$. The DNA bound water shows differences with respect to the water in bulk solution, such as partial ordering and lower mobility. The formation of the first hydration shells surrounding the DNA molecules gives rise the detected attractive forces (tensile stress) due to dipole-dipole interactions ${ }^{17-19}$. As the r.h. increases above 5-20\% (stage II), water molecules adsorb between the hydration shells (water II in cartoon of Fig. 1), initiating water percolation. The anchorage of the tightly packed ssDNA molecules via the thiol groups restricts the accommodation of the hydrogen bond network leading to an increasingly repulsive steric hindrance (compressive stress) ${ }^{17-19}$. Finally, in the third regime of water adsorption (stage III), the small slope of the surface stress curves found at r.h. $>60 \%$ strongly suggests the adsorption of water on top of the DNA films, once that the intermolecular channels have been filled up. A key signature of the response of the SsDNA monolayer is the hysteresis in the hydration/dehydration loop, which indicates that the process is accompanied by energy dissipation, suggesting the existence of capillary-like forces between the ssDNA molecules ${ }^{24}$. In fact, this signature has been found in nanoporous materials, indicating that our membrane model with intermolecular channels is a good approach to understand the phenomena ${ }^{25}$. During dehydration, only at very low humidity (r.h.<20\%) the available water molecules are not enough to maintain the capillary molecular bridges and the microcantilever tension returns to the initial value.

The next step was to hybridize the ssDNA-sensitized cantilever with the complementary ssDNA sequence (see Methods). Once the cantilever was hybridized, rinsed and dried, the hydration-induced tension of the microcantilever resulted in a radically different behaviour with respect to that of ssDNA (Fig. 2). First, the surface stress decreased with r.h. about $100-150 \mathrm{mN} / \mathrm{m}$ from the dehydrated to the fully hydrated state, i.e., the initial raise of the surface stress observed for the ssDNA case vanishes. This different behaviour constitutes a clear fingerprint of the hybridization 
process, and it will be used to monitor the performance of the biosensor. Secondly, the hysteresis in the hydration/dehydration loop is largely reduced upon hybridization. Neither longer hybridization times nor higher concentrations of the complementary target produce a significant change in the described cantilever response. Based on the slope of the curve, we can distinguish only two stages. In stage $I^{\star}$, there is a pronounced decrease of the surface stress between r.h. $\approx 0$ and r.h. $\approx 40 \%$. The surface stress variation is related to the steric hindrance to the water intercalation that arises from the blockade of the intermolecular channels by DNA duplexes formed in the biomembrane (see cartoon in Fig. 2). Notice that this phase is similar to stage II in the unhybridized ssDNA film (Fig. 1) that is governed by similar steric interactions. For higher humidity, stage ${ }^{*}{ }^{*}$, the slight decrease of the surface stress suggests adsorption of water on top of the nucleic acid monolayer as in stage III in the ssDNA film. The repulsive nature of the inter-DNA interactions upon hydration also leads to a large reduction in the hysteresis of the surface stress. In turn, when the sensitized cantilever was exposed to non-complementary DNA, the surface stress response to hydration showed no significant changes with respect to the non-hybridized sensitized cantilever (see Supplementary Information). This implies that specific Watson-Crick base pairing is the key intermolecular interaction that produces the observed differential phenomena described above.

To gain new insights into the hydration phenomena in DNA monolayers, we performed experiments in which SAMs of peptide nucleic acids (SSPNA) were formed on the gold-coated side of the cantilever. PNA is a synthetic mimic of the DNA in which the charged phosphate-sugar backbone is substituted by a peptidomimetic linear polymer, to which the nucleobases are linked in a conformation prone to specifically interact with complementary natural nucleic acids (DNA or RNA). Since the PNA is uncharged and the SAMs are formed in ultrapure water, these experiments shed light on the role of the electrostatic interactions in the DNA case. Moreover SAMs of PNA are known to exhibit a higher degree of order, with most of the molecules standing up $^{26,27}$. Fig. 3 shows the hydration/dehydration loops of surface stress for the SSPNA (a) and after subsequent hybridization with the complementary DNA (b). The surface stress response to the hydration retains all the qualitative basic features found for the DNA-sensitized cantilevers. This indicates that the electrostatic interactions do not play an essential role in the intermolecular interactions between nucleic acids and water molecules in highly packed films. These observations are consistent with the reported interactions between DNA molecules in solution ${ }^{17-19}$. For distances below $3 \mathrm{~nm}$, DNA molecules experience a large repulsive force as a consequence of the perturbation of the hydrogen-bond network surrounding the DNA molecules. The force, referred to as 
hydration force, exponentially increases with a $0.25-0.35 \mathrm{~nm}$ characteristic distance, and in consistency with our results, exhibits negligible dependence on the ionic strength. This indicates that for high humiditiy, the interaction between neighbouring DNA molecules on the metal surface could be governed by similar processes to those that occur in solution.

Figure $4 a$ shows the temporal evolution of the surface stress vs. r.h during hydration for a ssDNA monolayer exposed to $1 \mathrm{pM}$ of complementary DNA (dehydration curves were not depicted for the sake of simplicity). After six hours, equilibrium is achieved and the response is similar to that of $1 \mu \mathrm{M}$ for one hour, which indicates that the density of hybridized probes is similar in both cases. However for shorter incubation times, the interpretation of the surface stress vs r.h. curves are more complex. The most significant changes are produced at low humidity, r.h. below $40 \%$, referred to as stage I in Fig .2. For higher humidities (stage II) the surface stress variation is very similar in all curves. In particular, the initial variation of the surface stress in stage $I$ is very sensitive to the exposure time of the sensitized cantilever to the complementary DNA. This variation goes from positive values (tensile stress) to negative values (compressive stress). The surface stress response to the adsorption of the first water molecules can then be understood in terms of two competing interactions: the attractive forces driven by the hydrogen bonding (tensile stress) and the steric hindrance interactions (compressive stress). The dominant interaction is controlled by the size of the intermolecular channels and their blockade by the hybridized DNA molecules. In addition the curves exhibit tensile peaks at r.h.=30-40\%. These features observed at short incubation times and low humidity are actually beyond of our understanding. However, it is interesting to point out a number of processes that are not included in our phenomenological model that can contribute to variations of the surface stress. First, it is well-known that under limited hydration the structure of nucleic acids is different from the conformation under physiological conditions $^{22}$. The most studied transition is between the B- and A-forms of the doublestranded DNA that occurs at limited hydration, although other transitions can also occur. The $B$ helix is narrower and more extended than the $A$ counterpart and hence, in our system, the $A \rightarrow B$ transition as r.h. increases should produce a decrease of the repulsive intermolecular interactions between neighbouring molecules. Another factor that adds complexity to the observed phenomena is the slow hybridization kinetics in highly packed DNA monolayers ${ }^{28}$. In these films, steric crowding makes that only about $10 \%$ of the immobilised probes can form a duplex with the complementary sequence $^{12,29}$. In addition, the formation of the nucleation sites between the probe and 
the target prior the zipping reaction is significantly hindered, and it can last several hours. Thus, at intermediate times complementary ssDNA can be in two conformations: either completely zipped or joined to the ssDNA probe film via few nucleations points. The non-zipped complementary DNA can disrupt the structure of the monolayer, partially blocking the nanochannels to the intercalation of water molecules.

Despite the complexity of the detected phenomena, there is an intimate connection between the hydration-induced stress of the DNA SAM and the biomolecular interactions within. This opens the door to a novel label-free nucleic acid biosensor with high sensitivity. The most remarkable difference induced by the hybridization emerges at low r.h. during hydration, where the positive peak own to the ssDNA progressively vanishes with the degree of hybridization. Hence, we have calculated the area enclosed between the surface stress curves of the ssDNA film prior hybridization and after hybridization from r.h.=0 to r.h.=20\% (see inset graph in Fig. 2). The upper limit of r.h. $=20 \%$ has been chosen as an optimal value that includes the surface stress increase (stage I) in all curves recorded for SSDNA or SSPNA-sensitized cantilevers, and stage $I^{\star}$ after subsequent hybridization allowing a quick and unambiguous detection of the hybridization process. The calculated area is normalized by dividing by the area enclosed by curve of the ssDNA-sensitised cantilever before hybridization (see Methods). This parameter is referred to as 'sensor response', hereinafter. In Fig. 4b we plot the sensor response as a function of the hybridization time for a concentration of $1 \mathrm{pM}$ of complementary DNA.

In order to explore the specificity of the biosensor, we exposed ssDNA sensitised cantilevers to two kind of samples: i) a $1 \mu \mathrm{M}$ of ssDNA target with a single central mutation that originates a T/T mismatch (see Methods) and ii) a mixture of $1 \mathrm{nM}$ of complementary ssDNA and 1000-fold excess of non-matching ssDNA (Fig. 5). Fig. $5 a$ shows the surface stress vs. r.h. for a cantilever exposed to the target with the single mismatch for three hours. The surface stress response to hydration for $r$.h. $<20 \%$ is in between that of the SSDNA monolayer and that of the hybridization with the complementary target, although the initial tensile stress (stage I) is clearly observed. Since all the hybridization experiments were performed at $24^{\circ} \mathrm{C}$, significant variations are not expected in the hybridization yield of the fully complementary and singlemismatched sequence (the discriminatory temperature for this target is of about $43^{\circ} \mathrm{C}$ ). This indicates that the initial hydration-induced stress is sensitive to the difference in the duplex conformation as a consequence of the presence of a single mismatch. $A$ tentative explanation is that the initial tensile stress could arise from the stabilization of the mismatched bases in the duplex by hydrogen bonding with water molecules. This 
feature of the technique is relevant for genotyping applications contrarily to what happens in DNA microarrays and other current biosensors, the differential behaviour of the mismatched target does not rely on its tendency to de-hybridize at an optimized, fine-tuned working temperature (usually in the range $40-60^{\circ} \mathrm{C}$ ), but on its hydrationdependent response at room temperature. In turn, Fig. 5b shows the surface stress vs. r.h. when a cantilever is exposed to $1 \mathrm{nM}$ of complementary DNA in a background of 1 $\mu \mathrm{M}$ of non-complementary DNA. The hybridization curve is very similar to that obtained in a parallel experiment in which a sensitised cantilever was exposed to a pure solution with $1 \mu \mathrm{M}$ of complementary sequence and completely different to that obtained by exposing the sensitized cantilever to a pure solution with $1 \mu \mathrm{M}$ of non-complementary DNA. Therefore, this demonstrates that the technique has enough specificity for the discrimination of targets in complex mixtures, and, in particular, for the detection of minority genomes constituting only a $0.1 \%$ of the total amount of genomes in the sample. This resolution power points towards the use of the novel methodology in a variety of biotechnological and biomedical applications, including the detection and follow-up of minority genomes in RNA virus populations that can influence the evolution of the infected patient, as recently documented for human immunodeficiency virus in clinical samples.

As a summary of the sensitivity of the presented technique, we plot in Fig. $5 \mathrm{c}$ the value of the sensor response for a hybridization time of 3 hours, as a function of the concentration of the complementary target. We include the data for the mismatched sequence with a single $T / T$ central mismatch (shown above) and that obtained for a non-complementary DNA (negative control, see Supplementary Information). The sensor response is approximately constant for target concentrations higher than 0.5 $\mathrm{nM}$, showing that the sensitivity is at least in the picomolar range. The signal then decreases with the target concentration up to the analyzed value of $1 \mathrm{fM}$, which still remains about 2 times higher than the sensor response measured for the negative control. A key to obtain this extraordinary sensitivity is the high grafting density of the ssDNA monolayer that leaves sub-nanometer intermolecular channels in between. There are strong evidences that adsorption in nanoscale voids gives rise stresses that may exceed the surface stress of smooth surfaces by orders of magnitude ${ }^{6}$. Water intercalation within the nucleic acid film produces a significant stress whose pattern sensitively depends on the structure and chemistry of the monolayer. The hybridization process has an important impact in the collective properties of the nucleic acid film and in the capability of the molecular channels to bind water molecules ${ }^{27}$. The interactions involved in the hydration process, mainly steric and hydration forces, are of exponential 
nature, and then critically depend on variations in the conformation of the nucleic acid film.

The unprecedented sensitivity achieved by measuring the nanomechanical response of nucleic acids films upon hydration has not been reached by any biosensor able to detect unlabelled target samples. Detection of a single molecule is at the reach by miniaturization of the micro-scale cantilever to the nanoscale ${ }^{9,11}$. Previously, nanomechanical biosensors based on the surface-stress response have detected nucleic acids with high sensitivity in buffer solution, providing real-time information about the hybridization kinetics ${ }^{12,13}$. In those reports, a clear cantilever bending emerges after few minutes of interaction between the complementary sequences and the ssDNA probes on the cantilever. The hydration-based nanomechanical method proposed here does not provide real-time information and requires from one to three hours of incubation of the SSDNA or SSPNA-sensitised cantilevers with the nucleic acid sample. However, the hybridization provides an enormous quantitative and qualitative change in the hydration-induced tension of the nucleic acid film. In fact, the use of reference cantilevers is not necessary to detect hybridization, a practice that is essential for other in-situ measurements in order to remove the non-specific signals from fluctuations of the temperature and electrolyte concentration. We demonstrate a sensitivity up to the $\mathrm{fM}$ range, what means an enhancement of 3 orders of magnitude with respect to previous nanomechanical methods with similar cantilever sizes. More importantly, there is room for major improvements of the sensitivity and the throughput by measuring in parallel with hundreds of cantilevers with superior mechanical properties. These results, together with previous developments in nanomechanical sensors, make closer the use of this technology for genotyping and functional genomic research, allowing to tackle the early diagnosis of diseases by a straightforward method at room temperature, without the time-costly steps of amplification and labelling of the sample.

\section{METHODS}

\section{PREPARATION OF SSDNA SAMPLES}

ssDNA thiol-modified probes (with the 5' modification HS- $\left.\left(\mathrm{CH}_{2}\right)_{6}\right)$ and label-free ssDNA targets were obtained from Microsynth (Switzerland). DNA oligomers were HPLC purified and desiccated. Prior to use, the samples were resuspended in PBS buffer $\left(137 \mathrm{mM} \mathrm{NaCl}, 2.7 \mathrm{mM} \mathrm{KCl}, 8 \mathrm{mM} \mathrm{Na} \mathrm{HPO}_{4}, 2 \mathrm{mM}\right.$ $\mathrm{KH}_{2} \mathrm{PO}_{4} ; \mathrm{pH}=7.5$ ) and divided in aliquots of the desired volume and concentration without further modifications. All solutions were prepared using Milli-Q water $(18 \mathrm{M} \Omega / \mathrm{cm})$ and stored at $-20^{\circ} \mathrm{C}$. 
The oligonucleotide sequences used were the thiol-modified 16-mer probe 5'-HSCTACСTTTTTTTTCTG-3', the fully complementary target (5'-CAGAAAAAAAAGGTAG-3'), the target " $T$ " with a single mutation in its central region, that induces a T/T mismatch in the duplex (5'CAGAAAATAAAGGTAG-3') and a non complementary target used as negative hybridization control (5'-AGCTTCCGTACTCGAT-3').

\section{DNA IMMOBILIZATION AND HYBRIDIZATION}

Uncoated monocrystalline silicon microcantilever arrays were purchased from Mikromasch. Microcantilevers were $400 \mu \mathrm{m}$ long, $100 \mu \mathrm{m}$ wide and $0.6 \mu \mathrm{m}$ thick. The used cantilevers showed a resonance frequency of $5.3 \pm 0.1 \mathrm{kHz}$ and a spring constant (calculated by the Sader's method, ref. 30) of $0.029 \pm 0.001 \mathrm{~N} / \mathrm{m}$. The cantilevers were coated by e-beam evaporation with a $20 \mathrm{~nm}$ gold layer on top of a $2 \mathrm{~nm}$ adhesion layer of chromium at a deposition rate of $0.02 \mathrm{~nm} / \mathrm{s}$.

Freshly coated microcantilevers were incubated with $1 \mu \mathrm{M}$ of the ssDNA probe diluted in PBS at $24^{\circ} \mathrm{C}$, for 24-48 hours in order to immobilize a densely packed DNA layer that provides the hydration-induced surface stress curve shown in Fig. 1. Afterwards, the cantilevers were vigorously rinsed in PBS buffer and Milli-Q water to discard unspecific interactions. The duration of each washing was of about 20 minutes, and they were performed at the working temperature $\left(24^{\circ} \mathrm{C}\right)$. The cantilever were then dried under a stream of dry nitrogen gas. The hybridization of the sensitized cantilever with the target ssDNA was performed in PBS at $24^{\circ} \mathrm{C}$, at the desired target concentration and hybridization time. Afterwards, the cantilevers were rinsed and dried following the same protocol used for the sensitised cantilevers. To study the nanomechanical cantilever response as a function of the hybridization time (as in Fig. 4), the same cantilever was exposed to the target DNA for several times, followed by the steps of rinsing and drying described above.

\section{PNA IMMOBILIZATION}

A HPLC-purified ssPNA oligomer was used as the immobilized probe, with sequence (written from the amino to the carboxyl termini) Cys-O-O-AATCCCCGCAT (Applied Biosystems, Bedford, MA). Each “O” spacer unit is a molecule of 8-amino-3,6-dioxaoctanoic acid, used to separate the hybridization portion of the molecule from its 5' terminus. The overall length of spacer formed by two consecutive "O" linkers is $3.0 \mathrm{~nm}$. The terminal cysteine provides a thiol group that allows the interaction with gold, following a method previously described ${ }^{26}$. A ssPNA solution of $1 \mu \mathrm{M}$ was prepared in Milli-Q water (18 $\mathrm{M} \Omega / \mathrm{cm}$ ), where, contrarily to the natural nucleic acids, PNA remains active and functional. Immobilization of ssPNA on the cantilevers was performed at $24^{\circ} \mathrm{C}$ for $24 \mathrm{~h}$, by placing the chips in an Eppendorf tube containing a $10 \mu \mathrm{l}$ drop of the ssPNA solution. After immobilization, the chips were vigorously rinsed in $\mathrm{H}_{2} \mathrm{O}$ and finally dried in nitrogen gas for $30 \mathrm{~min}$. For hybridization experiments, the ssPNA-sensitized cantilever was explosed to the complementary ssDNA target (5'-ATGCGGGGATT3’). 
The nanomechanical response of the cantilevers was measured by using a home-built apparatus equipped with an environmental chamber $\left(\sim 400 \mathrm{~cm}^{3}\right)$ and an optical readout-technique that calculates the displacement of the cantilever at 30-50 positions along the cantilever longitudinal axis (Supplementary Information). The temperature was controlled by means of a Peltier cell placed below the cantilever, with a temperature sensor close to it. The relative humidity in the chamber was controlled by adjusting the flow rate between dry nitrogen and wet nitrogen (nitrogen bubbling through a wash flask filled with water) by means of precision valves. The total flow rate was of about $500 \mathrm{ml} / \mathrm{min}$. The relative humidity was changed at a rate of about $1 \%$ r.h. per minute. We have not found a significant dependence of the surface stress behaviour by varying this rate from 0.3 to $5 \%$ r.h. per minute. Prior to the measurement of the surface stress vs. r.h., the cantilevers were equilibrated at r.h. $=0 \%$ in a flow of dry nitrogen for one hour.

To calculate the surface stress, the cantilever profile was fitted with a second order polynomial to deduce the curvature radius. The surface stress $(\sigma)$ is related to the curvature radius $(R)$ by the Stoney's equation ${ }^{5}$,

$$
\sigma=\frac{E}{6(1-v)} \frac{T^{2}}{R}
$$

where $E=169 \mathrm{GPa}$ is the Young's modulus and $v=0.27$ is the Poisson coefficient of the silicon in the $<110>$ direction. The cantilever thickness $(\mathrm{T})$ was calculated from the measured value of the resonant frequency. The cantilever displacement was obtained by calibrating the position sensitive detector response (On-Trak Photonics, Inc.) to preset changes of angle between the cantilever and the detector (see Supplementary Information).

\section{SENSOR RESPONSE QUANTIFICATION}

The amount of hybridized probes is quantified by calculating the area enclosed between the immobilization and hybridization curves between r.h. $=0$ and r.h. $=20 \%$ (see graph inset in Fig. 2). This quantity is normalized by dividing by the area enclosed by the immobilization curve to minimize deviations due to variations in the mechanical properties of the cantilevers, gold coating and features of the ssDNA monolayer. The area enclosed by the curves is calculated by numerical integration of the experimental data. The processing of the data is described by the following equation;

$$
S R=\frac{\int_{0}^{20} d x\left[\sigma_{i m m o b}(x)-\sigma_{h y b}(x)\right]}{\int_{0}^{20} d x \sigma_{\text {immob }}(x)}
$$

Where $S R$ is the (adimensional) sensor response, $\sigma$ is the surface stress variation with the relative humidity, $x$ is the relative humidity and the subscripts immob and hyb denote the ssDNA sensitised cantilever and that cantilever after hybridization, respectively. The error bars in Figs. $4 \mathrm{~b}$ and $5 \mathrm{c}$ are 
calculated from the effect of the measurement error (measured from the signal fluctuations at a single humidity) on the sensor response (Eq. (2)).

Acknowledgements

D.R. acknowledges the fellowship funded by the Autonomic Community of Madrid (CAM). J.T, M.C, J.M and D.R acknowledge financial support by Spanish Ministry of Science (MEC) under grant No. TEC2006-10316 and CAM under grant No. 200550M056. C.B. acknowledges funding provided by MEC under grant No. BIO2007-67523. Work at Centro de Astrobiología was supported by European Union (EU), Instituto Nacional de Técnica Aeroespacial (INTA), MEC and CAM. All the authors acknowledge A. Cebollada, J.M. García-Martín, J. García, J.L. Costa-Kramer, M. Arroyo-Hernández and J.V. Anguita for their assistance in the gold deposition on the cantilevers.

Author contribution

J.T., J.M. and M.C. designed the hydration experiments. J.T., J.M, D.R and M.C. developed the measurement instrument. C.B, J.A.M-G and C.R. designed the PNA experiments for ruling out the effect of electrostatic forces. J.M., M.C. and C.R. carried out the experiments. All authors analysed the data. J.T., M.C., C.B. and J.A.M-G wrote the manuscript and all authors proof read it.

\section{References}

1. Israelachvili, J. \& Wennerstrom, H. Role of hydration and water structure in biological and colloidal interactions. Nature 379, 219-225 (1996).

2. Petrovykh, D.Y., Pérez-Dieste, V., Opdahl, A., Kimura-Suda, H., Sullivan, J.M., Tarlov, M.J., Himpsel, F.J. \& Whitman, L.J. Nucleobase orientation and ordering in films of single-stranded DNA on gold. J. Am. Chem. Soc. 128, 2-3 (2006).

3. Petrovykh, D.Y., Kimura-Suda, H., Whitman, L.J. \& Tarlov, M.J. Quantitative analysis and characterization of DNA immobilized on gold. J. Am. Chem. Soc. 125, 5219-5226 (2003).

4. Shu, W., McKendry, R.A., Watari, M., Riener, C.K., Balasubramanian, S., Welland, M.E. \& Strunz, T., DNA Molecular Motor Driven Micromechanical Cantilever Arrays. J. Am. Chem. Soc. 127, 17054-17060 (2005).

5. Thundat, T. Wachter, E. A., Sharp, S.L. \& Warmack R.J. Detection of mercury vapor using resonating microcantilevers. Appl. Phys. Lett. 66, 1695-1697 (1995).

6. Lavrik, N.V., Sepaniak, M.J. \& Datskos, P.G. Cantilever transducers as a platform for chemical and biological sensors. Rev. Sci. Instrum. 75, 2229-2253 (2004).

7. Raiteri, R., M. Grattarola, H.-J. Butt \& Skládal, P. Micromechanical cantilever-based biosensors. Sensors and Actuators B 79, 115-126 (2001).

8. Burg, T.P., Godin M., Knudsen, S.M., Shen, W., Carlson G., Foster, J.S., Babcok K., Mannalis S.R., Weighing of biomolecules, single cells and single nanoparticles in fluid. Nature 446, 1066-1069 (2007).

9. Li, M., Tang, H.X. \& Roukes, M.L. Ultra-sensitive NEMS-based cantilevers for sensing, scanned probe and very high-frequency applications. Nature Nanotechnology 2, 114120 (2007).

10. Gupta, A., Akin D. \& Bashir, R. Single virus particle mass detection using microresonators with nanoscale thickness. Appl. Phys. Lett. 84, 1976-1978 (2004). 
11. Ilic, B., Yang, Y., Aubin, K., Reichenbach, R., Krylov, S. \& Craighead, H.G. Enumeration of DNA Molecules Bound to a Nanomechanical Oscillator. Nano Letters 5, 925-929 (2005).

12. McKendry, R., Zhang, J., Arntz, Y., Strunz, T., Hegner, M., Lang, H.P., Baller, M.K., Certa, U., Meyer, E., Güntherodt, H.J., \& Gerber, C. Multiple label-free biodetection and quantitative DNA-binding assays on a nanomechanical cantilever array. Proc. Natl. Acad. Sci. USA 99, 9783-9788 (2002).

13. Zhang, J., Lang, H. P., Bietsch, A., Huber, F., Certa, U., Güntherodt, H.-J., Hegner, M. \& Gerber, Ch. Rapid and label-free nanomechanical detection of biomarker transcripts in human RNA Nature Nanotechnology, 1, 214-220 (2006).

14. Wu, G., Datar, R.H., Hansen, K.M., Thundat, T., Cote, R.J. \& Majumdar, A. Bioassay of prostate specific antigen (PSA) using microcantilevers. Nature Biotechnology 19, 856860 (2001).

15. Calleja, M., Tamayo J., Nordstrom, M. \& Boisen, A. Low noise polymeric nanomechanical biosensors. Appl. Phys. Lett. 88, 113901 (2006).

16. Bumbu, G.G., Wolkenhauer, M., Kircher, G., Gutmann, J.S. \& Berger R. Micromechanical Cantilever Technique a Tool for Investigating the Swelling of Polymer Brushes. Langmuir 23, 2203-2207 (2007).

17. Reddy, M.R. \& Berkowitz, M. Hydration forces between parallel DNA double helices: Computer simulations. Proc. Natl. Acad. Sci. USA 86, 3165-3168 (1989).

18. Rau, D.C., Lee, B., Parsegian, V.A. Measurement of the repulsive force between polyelectrolyte molecules in ionic solution: Hydration forces between parallel DNA double helices. Proc. Natl. Acad. Sci. USA 81, 2621-2625 (1984).

19. Hagan, M.F., Majumdar, A. \& Chakraborty, A.K. Nanomechanical forces generated by surface grafted DNA. J. Phys. Chem. B 106, 10163-10173 (2002).

20. Antoniou A. et al. Average risks of breast and ovarian cancer associated with BRCA1 or BRCA2 mutations detected in case Series unselected for family history: a combined analysis of 22 studies. Am J Hum Genet 72, 1117-1130 (2003).

21. Mertens, J., Álvarez, M. \& Tamayo, J. Real-time profile of microcantilevers for sensing applications. Appl. Phys. Lett. 87, 234102 (2005).

22. Tao, N.J., Lindsay, S.M. \& Rupprecht, A. Structure of the DNA Hydration Shells Studied by Raman Spectroscopy. Biopolymers 28, 1019-1030 (1989).

23. Schneider, B. \& Berman, H.M. Hydration of the DNA bases is local. Biophys. J. 69, 2661-2669 (1995).

24. Bryan, W.P. Thermodynamics of water-biopolymer interactions: irreversible sorption by a single uniform sorbent phase. Biopolymers 25, 1967-1979 (1986).

25. Casanova, F., Chiang, C.E., Li, C.-P. \& Schuller, I.K. Direct observation of cooperative effects in capillary condensation: the hysteretic origin. Appl. Phys. Lett. 91, 243103 (2007).

26. Briones, C., Mateo-Martí, E., Gómez-Navarro, C., Parro, V., Román, E. \& Martín-Gago, J.A. Ordered self-assembled monolayers of peptide nucleic acids with DNA recognition capability. Phys. Rev. Lett. 93, 208103 (2004).

27. Mateo-Martí, E., Briones, C., Pradier, C.M. \& Martín-Gago J.A. A DNA biosensor based on peptide nucleic acids on gold surfaces. Biosens. Bioelectron. 22, 1926-1932 (2007).

28. Hagan, M.F. \& Chakraborty, A.K. Hybridization dynamics of surface immobilized DNA. J. Chem. Phys. 120, 4958-4968 (2004).

29. Peterson, A.W., Heaton, R.J. \& Georgiadis, R.M. The effect of surface probe density on DNA hybridization. Nucleic Acids Research 29, 5163-5168 (2001).

30. Sader, J.E. Calibration of rectangular atomic force microscope cantilever, Rev. Sci. Instrum. 70, 3967-3969 (1999). 
Figure 1 Hydration dependence of the surface stress of highly-packed selfassembled ssDNA monolayers. The graph shows the surface stress variation during a hydration and dehydration cycle for a gold-coated silicon cantilever sensitised with a thiol-modified 16-mer ssDNA probe (symbols). The cantilever was incubated with a 1 $\mu \mathrm{M}$ solution of the ssDNA diluted in PBS at $24^{\circ} \mathrm{C}$ for $24 \mathrm{~h}$. The cantilever was then rinsed in PBS buffer and Milli-Q water at $24^{\circ} \mathrm{C}$ to discard unspecific interactions, and then dried under a stream of dry nitrogen gas. For comparison, the hydration/dehydration loop for the gold-coated cantilever previous to the functionalization is also shown (dashed lines). The surface stress variations are taken with respect to the surface stress at r.h. $=0$. The r.h. was changed at a rate of about $1 \%$ per minute. The formation of a highly packed SAM of SSDNA on the gold gives rise a significant and characteristic surface stress dependence on r.h., which is also accompanied of hysteresis in the hydration/dehydration loop. This dependence is not observed on the gold-coated cantilever. The surface stress change upon hydration is characterized by three stages. Cartoons of the ssDNA monolayer in stage I and stage II are also shown (right). The ssDNA molecules stand-up anchored to the gold via the thiol group. The intermolecular channels between ssDNA molecules host the adsorption of few water shells around the DNA molecules. During stage I, the adsorption of the first water molecules ('water l') gives a rapid increase of the surface stress (tensile stress). This arises from the increase of attractive forces provided by the water molecules bound to the ssDNA chains that build the hydration shell. In stage II, the population of water molecules in the intermolecular channels ('water II') leads to a repulsive hydration pressure that translates into a compressive change of the surface stress. In stage III, the surface stress slightly decreases with r.h. This stage is related to the adsorption of water on top of the DNA films.

Figure 2 Effect of hybridization on the surface stress vs. r.h. The graph shows the surface stress during a hydration/dehydration cycle for the ssDNA sensitised cantilever shown in Fig. 1, after exposure to $1 \mu \mathrm{M}$ of the complementary ssDNA target for one hour (symbols). For the purpose of this study, the surface stress at r.h. $\approx 0$ is chosen as the zero reference. Based on the slope of the surface stress upon hydration, two stages are distinguished. In stage $I^{*}$, the surface stress significantly decreases between r.h. $\approx 0$ and r.h. $\approx 40 \%$ as a consequence of the shrink of the intermolecular channels 
produced by the formed DNA duplexes. The water adsorption on the narrow channels leads to repulsive steric forces. For higher humidity, stage $\mathrm{II}^{\star}$, the slight decrease of the surface stress with r.h. is related to the adsorption of water on top of the nucleic acid monolayer. A cartoon of the DNA monolayer before (ssDNA) and after (dsDNA) hybridization during the initial hydration is shown at the right. The attractive forces induced by the hydration between the surface-grafted ssDNA molecules (Fig. 1) turn into repulsive after hybridization. This different behaviour constitutes the fingerprint of DNA-DNA hybridization and it is used to monitor the performance of the biosensor. Thus, the graph inset shows the surface stress vs. r.h. during the initial hydration for the ssDNA film (shown in Fig. 1) and after subsequent hybridization. The hybridization is quantified by the area enclosed by the curves between r.h. $=0$ and r.h. $=20 \%$ (dashed area).

Figure 3 Role of the electrostatic interactions: immobilization of SSPNA probes. Surface stress variation during hydration and dehydration cycles for a Cys-terminated 11-mer sSPNA probe immobilized on a gold-coated silicon cantilever (a), and after exposure to $1 \mu \mathrm{M}$ of the complementary target ssDNA (b). The immobilization was performed with $1 \mu \mathrm{M}$ of the ssPNA probe diluted in Milli-Q water at $24^{\circ} \mathrm{C}$ for $24 \mathrm{~h}$. The cantilever was then rinsed with Milli-Q water at $24^{\circ} \mathrm{C}$ to discard unspecific interactions, and then dried under a stream of dry nitrogen gas. The curves for the ssDNA film (Fig. 1) and subsequent DNA/DNA hybridization (Fig. 2) are also shown for comparison. Whereas DNA is a strongly charged molecule with an effective density of one fundamental negative charge per $0.17 \mathrm{~nm}$ of its length, PNA is an uncharged DNA mimic where the phosphate-sugar backbone has been replaced by a peptidomimetic polymeric structure. In addition, ssPNA forms highly packed SAMs on gold in ultrapure water without the need of ions, while ssDNA SAMs need to be formed in buffer solution. Although the curves exhibit significant quantitative differences showing a higher response of the PNA-based biosensor, the similar shape and the presence of hysteresis in both SSDNA and SSPNA monolayers, indicate that the electrostatic forces are not an essential element in the observed phenomena.

Figure 4 Hydration-induced surface stress as a function of the hybridization time. a, Surface stress vs. the r.h. for a ssDNA sensitised cantilever exposed to a solution of $1 \mathrm{pM}$ complementary ssDNA, as a function of the hybridization time. The experiment was performed by hybridizing, rinsing and drying the cantilever at different times. The 
response saturates for times longer than 6 h. b, Sensor response (calculated as defined in Methods) vs. hybridization time.

Figure 5 Specificity ( $a$ and b) and sensitivity (c) of the hydration-based DNA nanomechanosensor. $\mathbf{a}$, The effect of a nucleotide mutation in the target ssDNA on the hydration-induced surface stress (symbols). The mismatched sequence promotes a single $T / T$ central mismatch upon hybridization. The curve for the non hybridized ssDNA-sensitized biosensor (green), and that of the hybridization with the fully complementary sequence (red) are also shown for comparison. In the hybridization curves, the target concentration was $1 \mu \mathrm{M}$ and the hybridization time 3 hours. $\mathbf{b}$, Surface stress upon hydration of a sSDNA sensitised cantilever exposed to $1 \mathrm{nM}$ of complementary target in the presence of $1 \mu \mathrm{M}$ of non-complementary DNA. The curve for the non hybridized ssDNA-sensitized cantilever (green) and that of the hybridization with $1 \mu \mathrm{M}$ of the fully complementary sequence (red) are shown. The hybridization time was three hours. c, A plot of the sensor response (as defined in the text) as a function of the concentration of the complementary target ssDNA. The sensor response given by hybridization with non-complementary sSDNA (negative control) is also plotted and used to indicate the floor lever of sensitivity. The sensor response for the mismatched sequence at $1 \mu \mathrm{M}$ (derived from panel a) is also shown. 


\section{Figure 1}
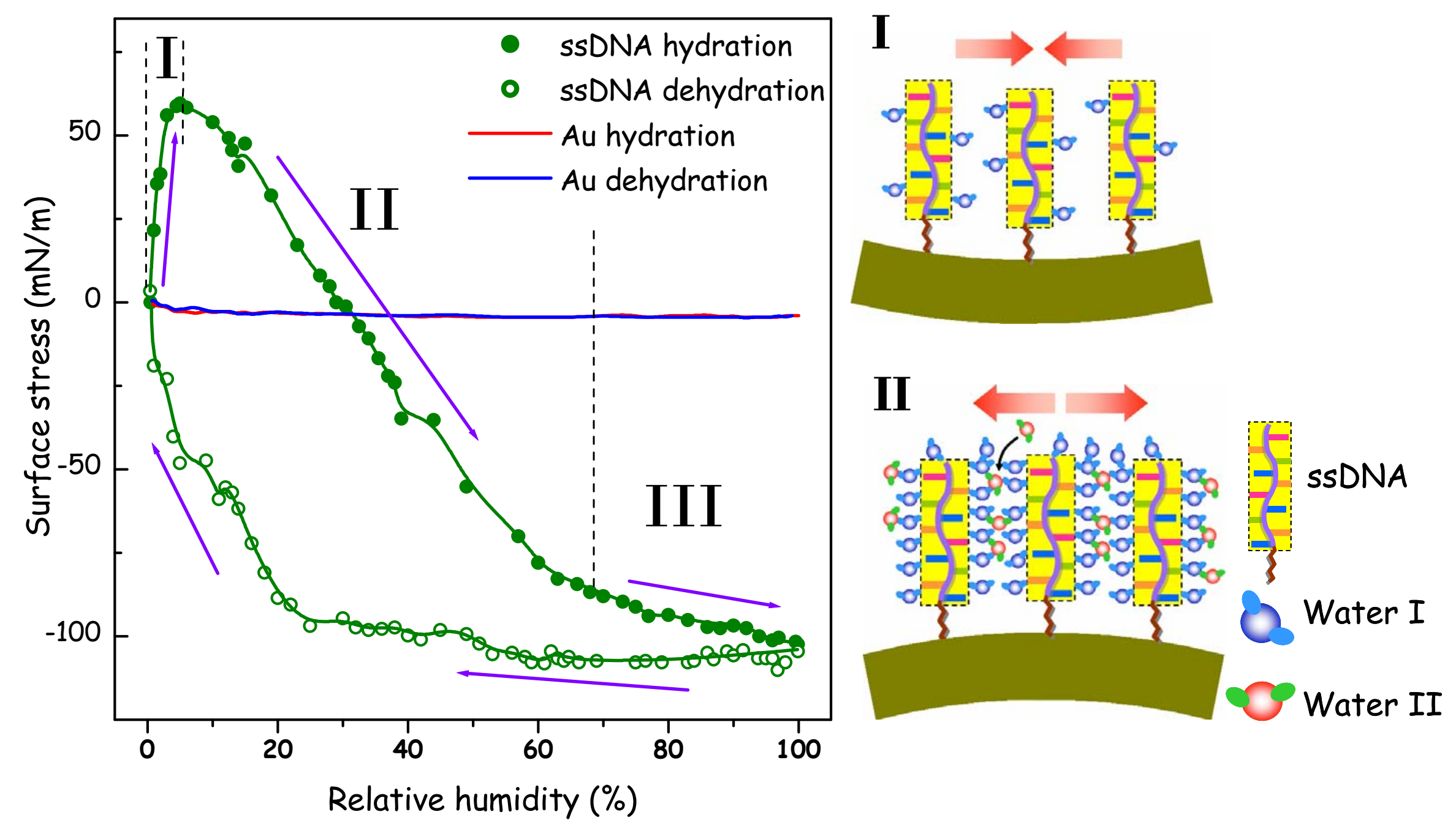


\section{Figure 2}
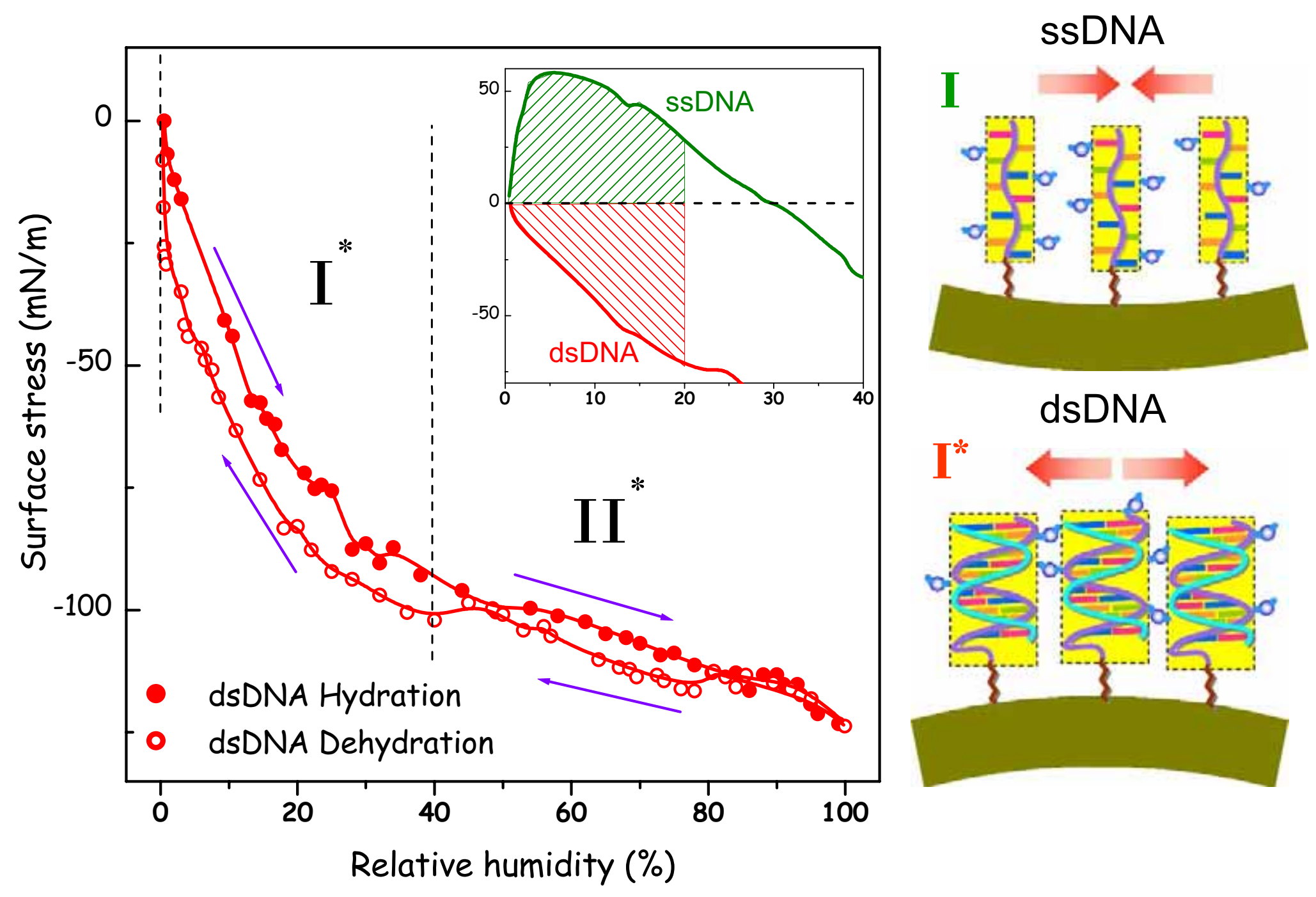


\section{Figure 3}

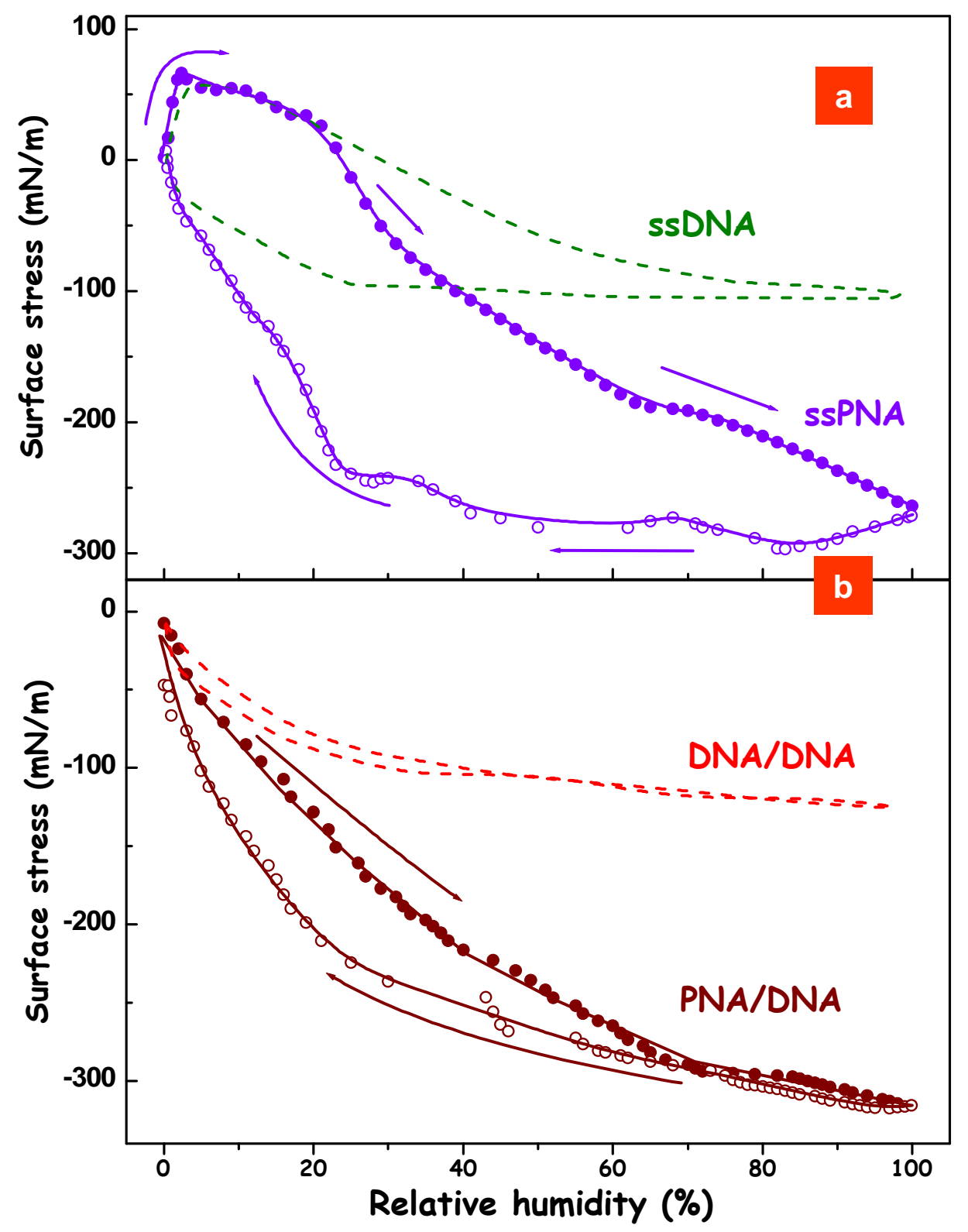




\section{Figure 4}
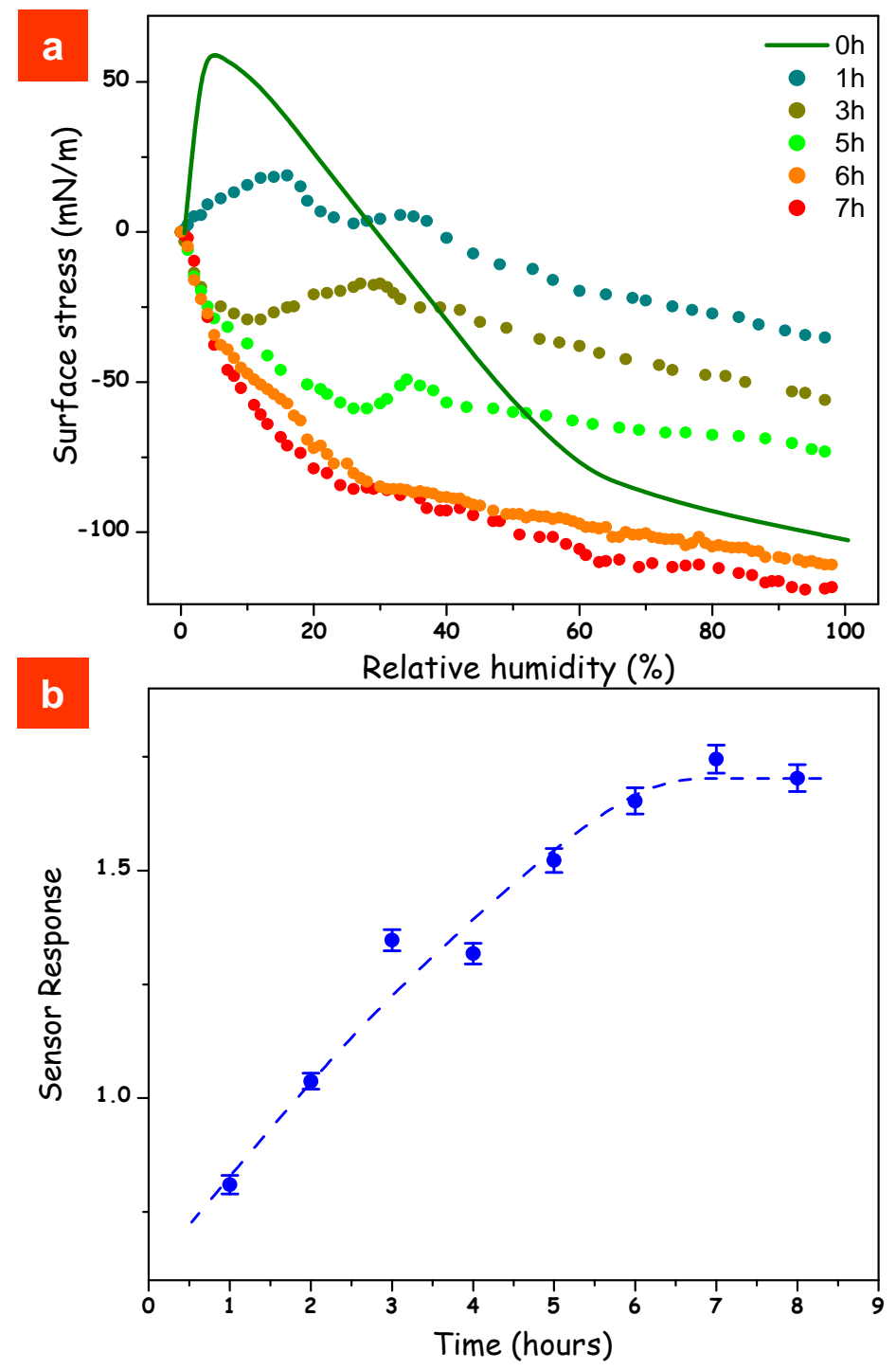

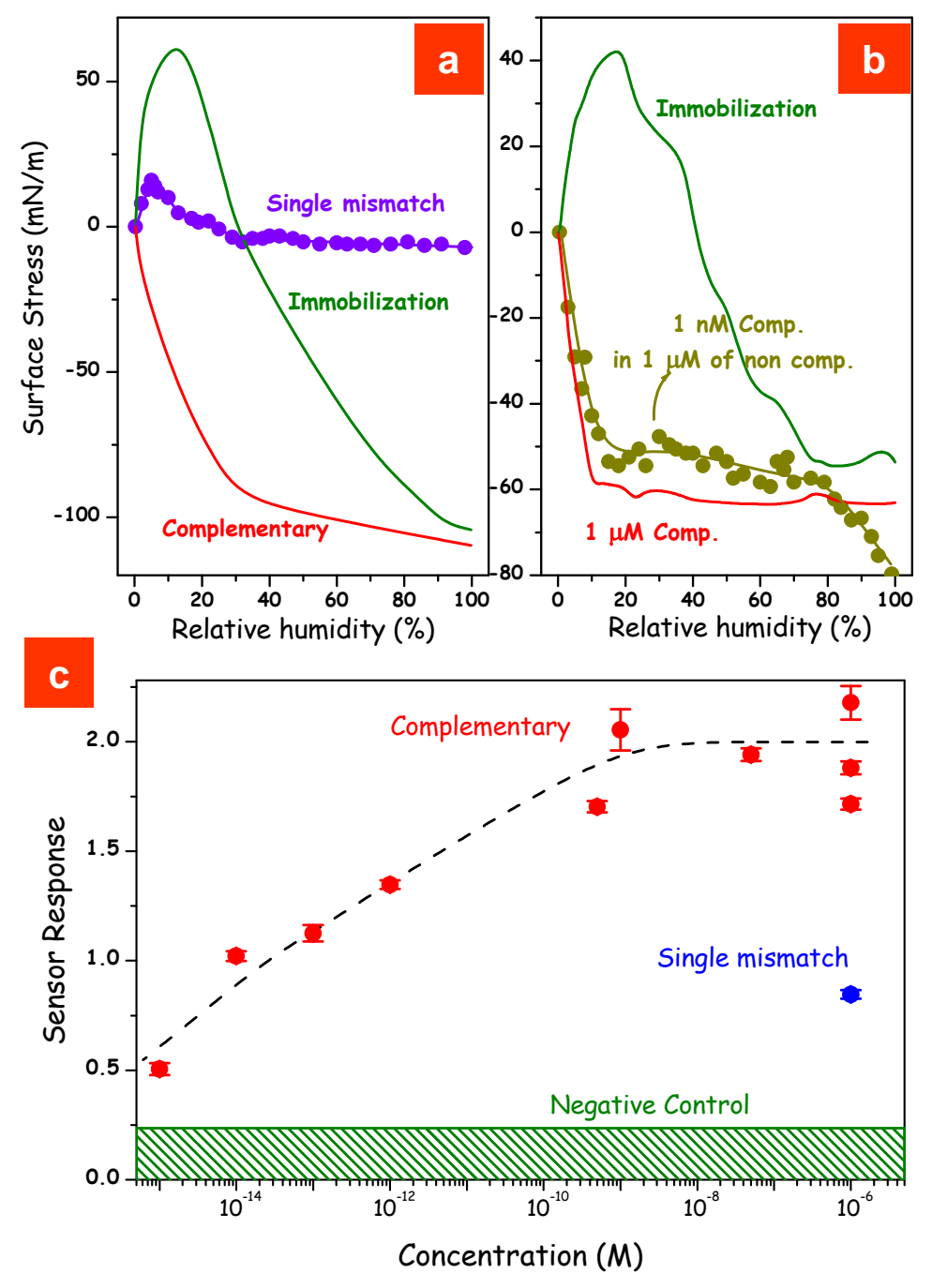